\title{
Immune memory in the brain
}

The brain's resident immune cells retain a long-lasting memory of peripheral inflammation. This memory can influence the response to stroke and the progression of Alzheimer's disease in mouse models. SEE ARTICLE P.332

\section{ALEXI NOTT \& CHRISTOPHER K. GLASS}

$\mathrm{T}$ The body's innate immune system provides an immediate and nonspecific defence against invading pathogens. The main innate immune cells in the brain, microglia, rarely encounter such infections, but they can respond to peripheral inflammation elsewhere in the body. One intriguing feature of peripheral innate immunity is a phenomenon called immune memory: the body's innate immune system 'remembers' previous exposures to microbes, and augments its responses to reinfections accordingly ${ }^{1,2}$. Does it follow, then, that peripheral inflammation could elicit immune memory in microglia? On page 332 , Wendeln et al. ${ }^{3}$ provide evidence for immune memory in microglia, and show that it can alter the progression of brain disorders in mice.

There are two types of immune memory: training, which exaggerates immune responses; and tolerance, which dampens them. To test whether microglia retain an immune memory of peripheral infection, Wendeln et al. injected lipopolysaccharide (LPS) molecules, a component of some bacteria, into the body cavities of mice. Exposing wild-type mice to two injections of LPS induced a microglial response resembling immune training, characterized by elevated levels of pro-inflammatory molecules. Four LPS exposures resulted in immune tolerance, indicated by reduced pro-inflammatory signals.

Peripheral immune training improves the immune system's ability to eliminate reinfections, but it can be harmful in people who have inflammatory conditions. By contrast, peripheral immune tolerance can be undesirable for clearing reinfections, but it is beneficial in organs that are subject to continuous pathogen exposure, such as the gut ${ }^{2}$. Changes in innate immunity in the brain are implicated in a diverse spectrum of disorders, but the contribution of immune memory to these has not been explored. Wendeln et al. therefore investigated whether immune training or tolerance in microglia could influence the progression of Alzheimer's disease and stroke, using mouse models.

A hallmark of Alzheimer's disease is the build-up of amyloid- $\beta$ protein, which activates
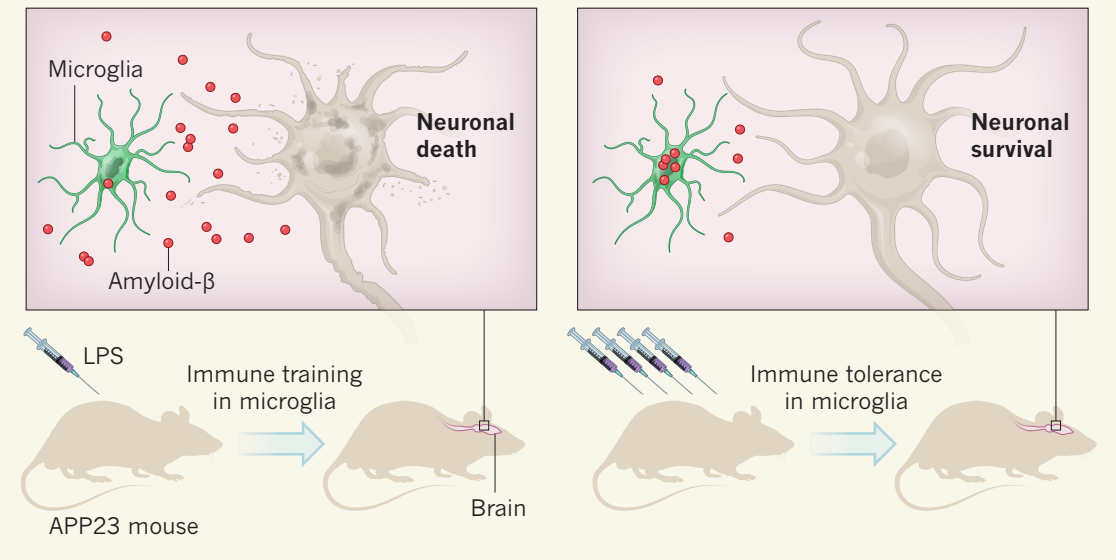

Figure 1 | Immune memory in a model of Alzheimer's disease. Innate immune cells can hold a 'memory' of previous exposure to pathogens that alters future immune responses. Wendeln et al. ${ }^{3}$ investigated innate immune memory in a mouse model of Alzheimer's disease (a strain dubbed APP23), in which the protein amyloid- $\beta$ accumulates in the brain. The authors injected lipopolysaccharide (LPS) molecules into the animals' body cavities either once or four times, to mimic bacterial infection. In mice that were injected once, brain immune cells called microglia exhibit a form of immune memory called immune training, which exaggerates inflammatory responses. After six months, amyloid- $\beta$ levels are higher than those in uninjected mice, and more neurons die. By contrast, the microglia of mice injected four times exhibit immune tolerance, which dampens inflammatory responses. This treatment leads to an increase in microglial amyloid- $\beta$ uptake and better neuronal survival.

microglia. The authors used a mouse model of Alzheimer's disease (a strain dubbed APP23) that recapitulates amyloid- $\beta$ accumulation in the brain. In this model, a single dose of LPS could induce microglial training, probably because the presence of amyloid- $\beta$ provided an additional pro-inflammatory stimulus. Four doses of LPS induced a tolerant response (Fig. 1). The researchers analysed the animals' brains after six months.

Wendeln and colleagues found that immune training increased amyloid- $\beta$ accumulation and that immune tolerance decreased amyloid- $\beta$, compared to levels in untreated animals of this strain. Similarly, immune tolerance reduced levels of neuronal death seven days after the researchers induced stroke in wild-type mice.

Wendeln et al. found that microglial immune memory persisted in APP23 mice for at least six months. Peripheral immune memory seems to be wired into stem cells, and so can be propagated in the cells' progeny for long time periods $s^{6-8}$. However, there is no evidence for the existence of microglial stem cells. Instead, immune memory in microglia might endure because these cells are longlived ${ }^{9}$. And persistent brain inflammation, initiated by amyloid- $\beta$ accumulation, could help to maintain immune memory.

What mechanisms might sustain microglial immune memory? Regions of DNA called enhancers can boost the expression of nearby genes when activated, thereby controlling cell behaviour. Enhancers in bone-marrowderived immune cells can become activated or poised for activation following transient peripheral inflammation and can persist in this state after inflammation ceases, providing a 'memory' of past events ${ }^{10}$. Wendeln et al. showed that treating APP23 mice with LPS increased levels of enhancer activation in microglia, compared to treating healthy mice with LPS. This indicates that long-lasting enhancer activation in microglia requires both brain pathology and an inflammatory insult.

The researchers demonstrated that microglial tolerance led to enhancer and geneexpression changes associated with a process called phagocytosis, by which microglia 
ingest material to be broken down. These changes correlated with increased microglial uptake of amyloid- $\beta$. By contrast, training led to enhancer and gene-expression changes associated with inflammation and energy expenditure.

Could targeting enhancer activity be a way of regulating immune memory in the brain, thereby altering the progress of neurological disorders? Enhancer activation is modulated by histone deacetylase (HDAC) enzymes, and the authors found that the loss of HDAC2 in microglia blocked immune training in these cells in healthy mice. Reduction of HDAC2 levels in neurons has been shown to improve memory and reverse gene-expression changes induced by neurodegeneration ${ }^{11}$. Furthermore, loss of $\mathrm{HDAC} 1$ and HDAC2 in microglia reduces amyloid- $\beta$ levels and improves memory in a different mouse model of Alzheimer's disease ${ }^{12}$. An appealing possibility is that HDAC2 inhibition blocks microglial immune training and enhances the cells' ability to clear amyloid- $\beta$ in people with Alzheimer's disease. However, regulating immune memory in the brain without causing deleterious consequences in the rest of the body will be challenging.

This work also opens up other avenues for research. For instance, metabolic products are often required for the activity of enzymes that regulate enhancers. Perhaps changes in metabolism mediate and perpetuate enhancer activation in microglia. Whether this is the case, and how specific enhancers are then targeted for training or tolerance, remains unknown.

The mechanism by which immune memory is transmitted to the brain from the periphery is also unknown. One possibility, suggested by Wendeln et al., is that inflammatory molecules are transported to the brain through the blood. Alternatively, peripheral immune cells might infiltrate the brain and activate microglia, or peripheral nerves might send signals to indicate that inflammation has occurred. One such possible neuronal pathway is the gut-brain axis, through which gut microbes can modulate microglial behaviour ${ }^{13}$.

The authors focused on microglia, but other cell types in the brain probably also contribute to the effects of training and tolerance. For example, cells called astrocytes, which have immunomodulatory functions, are activated during Alzheimer's disease ${ }^{14}$. The researchers showed that there were fewer activated astrocytes in APP23 mice exposed to either one or four doses of LPS than there were in untreated APP23 mice. This difference might be driven by microglia-derived factors ${ }^{15}$ - but another explanation is that astrocytes, which can detect inflammatory signals ${ }^{14}$, also retain a memory of previous stimuli. These possibilities still need to be tested.

Although rapid progress has been made in identifying genetic contributions to neurological disorders, environmental factors also have substantial effects. Because of this, Wendeln and co-workers' findings will be of broad interest. In addition to changes due to infections, the innate immune system can be influenced by environmental stimuli such as stress. Stress has been linked to neurological disorders, and an effect of stress on innate immune training has been hypothesized but remains unexplored in the context of these diseases ${ }^{16}$.

Finally, blocking immune training or mimicking immune tolerance in the brain will be of therapeutic benefit only if the current findings are replicated in humans. Demonstrating microglial immune memory in humans will be difficult, owing to the inaccessibility of brain tissue in living people. However, analysis of inflammatory signalling molecules released into the cerebrospinal fluid could be used as a proxy for microglial immune memory. Regardless of the immediate therapeutic potential, Wendeln and colleagues' work sets the stage for further investigation of the impact of environmental factors on microglial function in neurodegenerative conditions. .

Alexi Nott and Christopher K. Glass are in the Department of Cellular and Molecular
Medicine, University of California, San Diego, San Diego, California 92093, USA. C.K.G. is also in the Department of Medicine, University of California, San Diego.

e-mail:ckg@ucsd.edu

1. Netea, M. G., Latz, E., Mills, K. H. G. \& O'Neill, L. A. J. Nature Immunol. 16, 675-679 (2015).

2. Netea, M. G. et al. Science 352, aaf1098 (2016).

3. Wendeln, A.-C. et al. Nature 556, 332-338 (2018).

4. Labzin, L. I., Heneka, M. T. \& Latz, E. Annu. Rev. Med. 69, 437-449 (2018).

5. Sturchler-Pierrat, C. et al. Proc. Natl Acad. Sci. USA 94, 13287-13292 (1997)

6. Kaufmann, E. et al. Cell 172, 176-190 (2018).

7. Mitroulis, l. et al. Cell 172, 147-161 (2018).

8. Christ, A. et al. Cell 172, 162-175 (2018).

9. Füger, P. et al. Nature Neurosci. 20, 1371-1376 (2017).

10.Ostuni, R. et al. Cell 152, 157-171 (2013).

11.Gräff, J. et al. Nature 483, 222-226 (2012)

12.Datta, M. et al. Immunity 48, 514-529 (2018).

13.Erny, D. et al. Nature Neurosci. 18, 965-977 (2015).

14.Sofroniew, M. V. Nature Rev. Neurosci. 16, 249-263 (2015).

15.Liddelow, S. A et al. Nature 541, 481-487 (2017).

16.Salam, A. P., Borsini, A. \& Zunszain, P. A.

Mol. Psychiatry 23, 170-176 (2018).

This article was published online on 11 April 2018.

\section{NANOSCIENCE}

\section{A peptide-guided twist of light}

The growth of gold nanoparticles has been manipulated using amino acids and peptides to produce twisted structures that alter the rotation of light. The method could simplify the development of optical devices. SEE LETTER P.360

\section{GUILLERMO GONZÁLEZ-RUBIO \& LUIS M. LIZ-MARZÁN}

$\mathrm{N}$ anoparticles that control the rotation of light have potential applications, for example in optical devices ${ }^{1}$ and sensors $^{2}$, but preparing such particles has been difficult, especially from crystalline metals. On page 360 , Lee et al. ${ }^{3}$ report a remarkable method that uses amino acids or peptides (small molecules formed from amino acids) to direct the dissymmetric growth of gold nanoparticles that have a twisted morphology. The findings open up radical opportunities for the preparation of materials and devices that control light rotation.

Dissymmetric objects that cannot be superimposed on their mirror image are found at a variety of scales and include molecules of DNA, snail shells and even galaxies. Such structures are said to be chiral. Louis Pasteur coined the concept of molecular dissymmetry in 1848 , when he attributed the morphological differences in crystals of tartrate to the existence of mirror-image tartrate molecules ${ }^{4,5}$. We now know that the functions of biomolecules often depend on chirality, which, for example, provides the basis of exquisitely specific interactions between enzymes and their substrate molecules, enabling the proper functioning of living organisms.

One property of chiral molecules is that each mirror-image form interacts differently with circularly polarized light (in which the electric field traces a helix in the direction of the light's propagation), resulting in phenomena known collectively as optical activity. For example, circular dichroism involves the differential absorption of left- and right-handed circularly polarized light by the mirror-image forms of a molecule. The optical activity of chiral organic molecules has been used to manipulate the rotation of light, but almost invariably in the ultraviolet region of the electromagnetic spectrum.

In the past decade, some inorganic materials have also been shown to have chirality and optical activity ${ }^{6}$, thereby enabling control of the rotary propagation of light to be extended to the visible and near-infrared regions. Prominent among these inorganic compounds are nanostructured materials that 